\title{
Crossing the Line: Care of a Pediatric Patient with Intractable Seizures and Severe Neuropathic Pain in Absence of Access to Medical Marijuana
}

\author{
Meaghann S. Weaver, MD, MPH, FAAP, ${ }^{1}$ Adam R. White, JD, MHA, ${ }^{2}$ and Jacob Robinson, $\mathrm{MS}^{1}$
}

\begin{abstract}
Background: A reality of the current political and legal environment is that while marijuana and cannabis-based products remain not approved for human consumption at the federal level in the United States, several states have authorized use for constituents. While state lines represent meaningful cultural and geographical identity markers, the reality is that patients and families readily cross state lines to access medical interventions and care.

Methods: We present the case of a six-year-old child with intractable seizures and severe neuropathic pain managed on medical marijuana (MM) in her home state of Colorado; where medicinal use of marijuana is authorized at the state level; traveling across state lines to access surgical care in Nebraska where MM is prohibited.

Conclusion: The case report shares the communication and creativity invested in adequate symptom management for this child weaned off of MM perioperatively. The case recognizes the unique complexities of shared symptom management goals within state-specific care models.
\end{abstract}

Keywords: cannabis; medical marijuana; pediatric palliative care; symptom management

\section{Introduction}

C ANNABINOID-BASED PRODUCTS are direct advertised to consumers over the Internet and in media circuits. In a recent review of nonprescription cannabinoid-based medicine use in Canadian hospices, a setting where marijuana remains a Schedule I drug, 35 (88\%) pediatric hospice organizations knew of families that use cannabinoid-based medicine to manage the symptoms of their child. ${ }^{1}$ For those geographies where medical marijuana ( $\mathrm{MM})$ is permitted, medical providers are asked with increasing frequency for access options. In a cross-sectional survey of 288 pediatric oncologists in Illinois, Massachusetts, and Washington (states with legalized $\mathrm{MM}$ ), more than one-third of the responding pediatric oncologists received $\mathrm{MM}$ requests in the month before survey completion with $92 \%$ of these providers willing to help pediatric cancer patients access $\mathrm{MM}^{2}$

Marijuana was available in pharmacies in the United States for pain and muscle spasm relief before the imposition of the Marijuana Tax Act of 1937. This heavy tax resulted in marijuana's removal from almost all pharmacies by 1941 . In 1996, California became the first state to "allow" MM in the United States under state law, despite the federal Controlled Substances Act's inclusion of marijuana as a Schedule I controlled substance in $1970 .^{3}$ As of May 2019, 33 states and the District of Columbia have enacted state laws making marijuana legal for medical and/or recreational purposes. 4

The Agricultural Improvement Act of 2018 (referred to as the "Farm Bill") became law in December of 2018, changing the Controlled Substances Act's definition of marijuana to remove industrial "hemp" from Schedule I listing. 5 Effectively, the Farm Bill legalized the use and possession of cannabis with a delta-9 tetrahydrocannabinol ("THC") concentration of not more than 0.3 percent $^{6}$ for purposes of the federal Controlled Substances Act. The changes brought about for rural communities by the Farm Bill, however, did not change Nebraska's definition of marijuana, which more closely follows the original definition of the federal Controlled Substances Act.*

Notwithstanding the changes to marijuana's legal status in many surrounding states, and the passage of the Farm Bill,

* Nebraska allows for use of "industrial hemp" only when grown and possessed by a postsecondary institution or the Department of Agriculture. Neb. Rev. Stat. §2-5701.

${ }^{1}$ Children's Hospital and Medical Center Omaha, Division of Pediatric Palliative Care, Omaha, Nebraska.

${ }^{2}$ Children's Hospital and Medical Center Omaha, Office of the General Counsel, Omaha, Nebraska.

Accepted May 17, 2019. 
the use of marijuana, and any other "material, compound, mixture, or preparation" containing marijuana or its isomers, is illegal in the State of Nebraska ${ }^{7}$ with two very limited exceptions. With the recent FDA classification of Epidiolex, cannabidiol ("CBD') manufactured by Greenwich Biosciences, Nebraska now has two limited circumstances in which a person may possess and use cannabinoids: Epidiolex may be used by prescription ${ }^{8}$; and, other CBD formulations may be used only if the patient is enrolled in the "Medical Cannabidiol Pilot Study.",9

\section{Case Description}

A six-year-old female with severe hypoxemic brain injury experienced medically-refractory daily seizures since early infancy. The child resided in her family's home state of Colorado where medicinal use of marijuana is authorized at the state level but was traveling to Nebraska for a surgical procedure where $\mathrm{MM}$ is prohibited. The child received blended whole food feeds with tablespoons of grounded MM added through gastrostomy tube thrice per day. She was on room air other than sometimes given nasal cannula oxygen "for comfort" during extended seizures based on family preference rather than strict pulse oximetry reading.

In preparation for traveling for a surgical intervention for seizure management available in Nebraska, the family reached out to the pediatric palliative care team for additional support during their anticipated two-week stay in Omaha. The family sent medical records, which included verification of "intractable seizures and neuropathic pain" from the child's primary neurologist in Colorado. The medical records shared included correspondence from a marijuana dispensary in Colorado citing weekly allocation of MM by grams.

On further communication with the child's family, the family explained that she started MM eight months ago with noted decrease in her seizure frequency and duration. The child was not verbal, but the family shared that they perceived she had also experienced pain relief as her spasticity and seizure-tonicity were associated with nonverbal signs of discomfort. The family was surprised to hear that the receiving palliative care team was not able to administer MM in their neighboring state's pediatric hospital due to difference in state laws between Colorado and Nebraska.

\section{Discussion}

This case report shares the unique and challenging circumstance of complex symptom management for symptoms perceived to be improved by MM in a setting prohibitive of MM use. The receiving pediatric palliative care team was without clear knowledge of the child's consistent THC/CBD concentration or MM weaning protocols. The child's family gave consent for the receiving pediatric palliative care physician to contact the dispensary in her home state for clarity regarding THC/CBD concentration. The dispensary informed the physician that the dried cannabis product was from the ruderalis plant species but that the THC/CBD dosing was not consistently known per gram dispensed per week. In online CBD products with concentration markings, studies have shown that only onethird of products sold online are labeled accurately. ${ }^{10}$ Interestingly, the MM was provided to the family in a zip-baggie despite other young children residing in the home, which raised our pediatric team's concern for lack of childproof packing.
In preparation for the child's travels into the study team's home state, an extensive search was completed for open CBD or THC clinical trials, but without yield. The team thus worked together with the family to strategize a slow-wean in her home MM in preparation for symptom management using authorized pharmaceuticals and integrative therapies in the receiving state.

\section{Seizure Intervention}

The patient was on valproic acid and clobazam scheduled for seizures with intranasal midazolam available for rescue medication. The family added MM with hope for increased seizure control based on Internet reading. CBD has reported success as a seizure-frequency reducer. ${ }^{11}$ Many other offlabel investigations into medical effectiveness of CBD have demonstrated effectiveness. ${ }^{12-14} \mathrm{CBD}$, unlike its common cannabis-based cousin THC, does not elicit adverse physiological effects in up to 50 times recommended daily doses ${ }^{15}$ and has even been shown to counteract intoxicating effects of THC. ${ }^{16}$ Despite physiological effects being demonstrated through CBD usage, molecular mechanisms and the pharmacokinetic profile of $\mathrm{CBD}$ are much less understood than THC. ${ }^{17}$

The first FDA approved Cannabis-based drug product was released in 2018. The drug, named Epidiolex, is a CBD oral medication approved for usage in Lennox-Gastaut and Dravet syndrome patients two years or older. Randomized, controlled investigations into Epidiolex effectiveness in children with Dravet syndrome and Lennox-Gastaut syndrome demonstrated the drug's effectiveness in significantly reducing seizure frequency and duration compared to placebo cohorts. ${ }^{18,19}$

After communication with the child's neurologist in Colorado, the pediatric palliative care team obtained insurance authorization and access to Epidiolex for the patient with notable seizure control during her hospitalization in Nebraska. The primary neurologist committed to continuation of Epidiolex and clinical monitoring upon discharge back home to Colorado. The palliative care team, the home neurologist, and the child's family agreed that holding the MM while starting Epidiolex would be a meaningful way to monitor for seizure impact and side effects. The child's neurologist expressed surprise when informed of the quantity of MM the child was receiving before transfer to Nebraska. The neurologist shared that his primary role had been to provide written verification of her diagnosis, which the family brought to the dispensary, but that the dosing and access trends were monitored by the dispensary rather than his care team. In most recent follow-up communication with the child's mother, the family had decided to resume MM five weeks after returning home to Colorado due to their perception of "benefit in overall comfort on MM" but at one-fifth the prior MM dose with ongoing seizure control on Epidiolex.

The patient in our case study had historically been on clobazam for intractable seizure control. CBD increases clobazam levels and side effects in children with epilepsy. ${ }^{20}$ Sharing this information with the child's prescribing team and family was an important approach to transition to a safer antiepileptic in the setting of her concurrent MM use in her home setting. 


\section{Neuropathic Pain}

The patient's family described starting $\mathrm{MM}$ for pain management secondary to her spasticity and contractures. Neuropathic pain investigations for pediatric populations have not yet been established for THC or CBD. Cannabinoid receptors are present in brain regions associated with analgesia and on afferent nerves that respond to painful stimuli. ${ }^{21}$ Randomized controlled studies have demonstrated MM's effect in improved neuropathic pain patterns in adult populations with resultant improved quality of life. ${ }^{22,23}$ However, improved pain management correlates to an increased dosage, which in turn elicits an increase in many of the associated untoward side effects. ${ }^{24}$ The patient's family utilized $\mathrm{MM}$ as her first-line intervention for neuropathic pain. MM ideally is not considered first line for any pediatric condition. Our team worked with the family to add pharmaceuticals with less potential toxicity and better established pediatric evidence.

Due to history of spasticity/pain symptoms heightened in evening hours, the patient was started on gabapentin. A lowdose alpha-2-agonist (clonidine) was sequentially added to target the spasticity. The doses were weight based to avoid sedation/sleepiness as unwanted side effects. The family reported that their child appeared more comfortable with maintained sleep-wake cycles.

\section{Integrative Therapies}

When asked about the aspects of MM use that made the intervention most appealing or meaningful for the family, the child's family cited a desire for "what is natural." The receiving palliative care team thus worked together with the family to select essential oils and organic coconut oil carrier salve for foot reflexology and gentle pressure point application. The family benefited from selection of salt lamp lighting for the child's room and inclusion of Healing Touch, Massage Therapy, and passive Tai Chi movements in both the child and the parental caregivers' care. The family's desire to incorporate natural approach to care was honored in these safe ways during her hospitalization.

Of particular relevance for this child's family was the indication that MM can work independent of opioid treatments and may potentially serve as an alternative for pain management. ${ }^{25}$ The child's family felt surprised to hear that they were not able to continue an intervention that they had started to try to avoid what they termed "those other addicting medicines" in the midst of an opiate public health crisis. The family revealed that with a remote family history of opiate substance abuse disorder, there had been a family desire to avoid "addicting medications" for the child. This trustful disclosure opened opportunity for deep listening about the family's experience of prescribed pharmaceuticals having been misused in a way damaging to extended family relationships.

Conversation with the family provided opportunity for collaborative education on safe dosing, diversion prevention, and attentiveness to prior family memories while also fostering caring management of a child's pain burden.

\section{Summary}

The current state-specific approach to MM notably burdens patients, families, and health care systems with a fragmented approach to symptom management based on local context. The stigmatization or legal implications of MM in certain settings may lead well-meaning providers to avoid asking about use or to struggle with appropriate response. Provider response to parents reporting MM use in Schedule I settings notably varies from direct inquiry, feigned ignorance, or informed ignoring. ${ }^{1}$ Ideally, providers would compassionately and competently inquire about pharmaceutical and nonpharmaceutical interventions (to include MM use) as part of comprehensive palliative care symptom assessments. ${ }^{26}$

Our pediatric palliative care team opted for honesty, transparency, and proactive preparation in supporting this child and family through a transition into our care setting where MM is not legally administered and yet where the shared goal was to ensure excellent symptom management. Our inquiring offered meaningful opportunity to partner with the family for safe symptom management.

The American Academy of Pediatrics policy statement specifically opposes marijuana use in pediatric populations but recognizes "that marijuana may currently be an option for cannabinoid administration for children with life-limiting or severely debilitating conditions and for whom current therapies are inadequate." 27 Future studies to explore the efficacy and role of MM in pediatric palliative care settings are warranted and yet not feasible with current Schedule I drug status. This patient had experienced perinatal brain injury as a multifactorial and yet contributory etiology of her intractable seizures. Results from both pig and murine models have demonstrated decreased density of necrotic neurons and modulated cytokine release in perinatal hypoxemic injury in these animal models. ${ }^{28,29}$ Further research on MM has potential to advance understanding not just in basic science but also clinical translation for competent and compassionate intervention.

\section{Author Disclosure Statement}

No competing financial interests exist.

\section{References}

1. Tatterton MJ, Walker C: The prevalence of nonprescription cannabinoid-based medicines in british children's hospices: Results of a national survey. J Palliat Med 2019 [Epub ahead of print]; DOI: 10.1089/jpm.2018.0522.

2. Ananth P, Ma C, Al-Sayegh H, et al.: Provider perspectives on use of medical marijuana in children with cancer. Pediatrics 2018;141:e20170559.

3. Mead A: The legal status of cannabis (marijuana) and cannabidiol (cbd) under U.S. Law. Epilepsy Behav 2017;70: 288-291.

4. Mauro PM, Santaella-Tenorio J, Perlmutter AS, et al.: Correct knowledge of medical cannabis legal status in one's own state: Differences between adolescents and adults in the United States, 2004-2013. Addict Behav 2019;88:23-28.

5. Agricultural Improvement Act of 2018, $\S 12619$.

6. Agricultural Marketing Act of 1946, §297A(1).

7. Neb. Rev. Stat. §28-405, Schedule I, (c)(7).

8. Neb. Rev. Stat. $\S 28-405$, Schedule V, (d).

9. Neb. Rev. Stat. $\S \S 28-463$ to $28-469$.

10. Bonn-Miller MO, Loflin MJE, Thomas BF, et al.: Labeling accuracy of cannabidiol extracts sold online. JAMA 2017; 318:1708-1709.

11. Porter BE, Jacobson C: Report of a parent survey of cannabidiol-enriched cannabis use in pediatric treatmentresistant epilepsy. Epilepsy Behav 2013;29:574-577. 
12. Sekar K, Pack A: Epidiolex as adjunct therapy for treatment of refractory epilepsy: A comprehensive review with a focus on adverse effects 2019;8; Doi: 10.12688/ f1000research.16515.1.

13. Tzadok M, Uliel-Siboni S, Linder I, et al.: CBD-enriched medical cannabis for intractable pediatric epilepsy: The current Israeli experience. Seizure 2016;35:41.

14. Szaflarski JP, Bebin EM, Cutter G, et al.: Cannabidiol improves frequency and severity of seizures and reduces adverse events in an open-label add-on prospective study. Epilepsy Behav 2018;87:131-136.

15. Bergamaschi MM, Queiroz RH, Zuardi AW, et al.: Safety and side effects of cannabidiol, a cannabis sativa constituent. Curr Drug Saf 2011;6:237-249.

16. Pertwee RG: Ligands that target cannabinoid receptors in the brain: From THC to anandamide and beyond. Addict Biol 2008;13:147-159.

17. Leo A, Russo E, Elia M: Cannabidiol and epilepsy: Rationale and therapeutic potential. Pharmacol Res 2016;107: 85-92.

18. Devinsky O, Cross JH, Laux L, et al.: Trial of cannabidiol for drug-resistant seizures in the dravet syndrome. N Engl J Med 2017;376:2011-2020.

19. Devinsky O, Marsh E, Friedman D, et al.: Cannabidiol in patients with treatment-resistant epilepsy: An open-label interventional trial. Lancet Neurol 2016;15:270-278.

20. Geffrey AL, Pollack SF, Bruno PL, et al.: Drug-drug interaction between clobazam and cannabidiol in children with refractory epilepsy. Epilepsia 2015;56:1246-1251.

21. Nielsen S, Sabioni P, Trigo JM, et al.: Opioid-sparing effect of cannabinoids: A systematic review and meta-analysis. Neuropsychopharmacology 2017;42:1752-1765.

22. Ware MA, Wang T, Shapiro S, et al.: Smoked cannabis for chronic neuropathic pain: a randomized controlled trial. CMAJ 2010;182:E701.
23. Wilsey BL, Deutsch R, Samara E, et al.: A preliminary evaluation of the relationship of cannabinoid blood concentrations with the analgesic response to vaporized cannabis. J Pain Res 2016;9:587-598.

24. Andreae MH, Carter GM, Shaparin N, et al.: Inhaled cannabis for chronic neuropathic pain: A meta-analysis of individual patient data. J Pain 2015;16:1221-1232.

25. Bachhuber MA, Saloner B, Cunningham CO, et al.: Medical cannabis laws and opioid analgesic overdose mortality in the United States, 1999-2010. JAMA Intern Med 2014; 174:1668-1673.

26. Briscoe J, Kamal AH, Casarett DJ: Top ten tips palliative care clinicians should know about medical cannabis. J Palliat Med 2019;22:319-325.

27. Committee on Substance Abuse. American Academy of Pediatrics: The impact of marijuana policies on youth: Clinical, research, and legal update. Pediatrics 2015;135:584-587.

28. Pazos MR, Mohammed N, Lafuente H, et al.: Mechanisms of cannabidiol neuroprotection in hypoxic-ischemic newborn pigs: Role of $5 \mathrm{ht}(1 \mathrm{a})$ and cb2 receptors. Neuropharmacology 2013;71:282-291.

29. Castillo A, Tolon MR, Fernandez-Ruiz J, et al.: The neuroprotective effect of cannabidiol in an in vitro model of newborn hypoxic-ischemic brain damage in mice is mediated by $\operatorname{cb}(2)$ and adenosine receptors. Neurobiol Dis 2010; $37: 434-440$.

Address correspondence to: Meaghann S. Weaver, MD, MPH, FAAP Division of Pediatric Palliative Care Children's Hospital and Medical Center Omaha 8200 Dodge Street Omaha, NE 68114

E-mail: MeWeaver@childrensomaha.org 